\title{
A Pattern Matching Compiler for Multiple Target Languages
}

\author{
Pierre-Etienne Moreau ${ }^{1}$, Christophe Ringeissen ${ }^{1}$, and Marian Vittek ${ }^{2}$ \\ 1 LORIA-INRIA, 615, rue du Jardin Botanique, \\ BP 101, 54602 Villers-lès-Nancy Cedex France \\ \{moreau,ringeiss\} @loria.fr, elan.loria.fr/tom \\ 2 Institut of Informatica Mlynska dolina, \\ 84215 Bratislava, Slovakia \\ vittek@fmph.uniba.sk
}

\begin{abstract}
Many processes can be seen as transformations of tree-like data structures. In compiler construction, for example, we continuously manipulate trees and perform tree transformations. This paper introduces a pattern matching compiler (TOM): a set of primitives which add pattern matching facilities to imperative languages such as C, Java, or Eiffel. We show that this tool is extremely non-intrusive, lightweight and useful to implement tree transformations. It is also flexible enough to allow the reuse of existing data structures.
\end{abstract}

\section{Introduction}

For the compiler construction, there is an obvious need for programming transformation of structured documents like trees or terms: parse trees, abstract syntax trees (ASTs for short). In this paper, our aim is to present a tool which is particularly well-suited for programming various transformations on trees/terms. In the paper we will often talk about "term" instead of "tree" due to the oneto-one correspondence between these two notions. Our tool results from our experience on using existing programming languages and programming paradigms to implement transformations of terms.

In declarative (logic/functional) programming languages, we may find some built-in support to manipulate structured expressions or terms. For instance, in functional programming, a transformation can be conveniently implemented as a function declared by pattern matching [2,612, where a set of patterns represents the different forms of terms we are interested in. A pattern may contain variables (or holes) to schematize arbitrary terms. Given a term to transform, the execution mechanism consists in finding a pattern that matches the term. When a match is found, variables are initialized and the code related to the pattern is executed. Thanks to the mechanism of pattern matching, one can implement a transformation in a declarative way, thus reducing the risk to implement it in the wrong way.

For efficiency reasons, it may be interesting to implement similar tree-like transformations using (low-level) imperative programming languages for which

G. Hedin (Ed.): CC 2003, LNCS 2622, pp. 6176 2003.

(C) Springer-Verlag Berlin Heidelberg 2003 
efficient compilers exist. Unfortunately, in such languages, there are no built-in facilities to manipulate term structures and to perform pattern matching. There are two common solutions to this problem.

One possibility would be to enrich an existing imperative programming language with pattern matching facilities $[5 / 7 / 813]$. This hard-wired approach ties users to a specific programming language. The situation is thus little better than that in declarative languages. Furthermore, because terms are built-in, user-defined data structures must be converted to the term structure. Such marshalling complicates the user's program, and it incurs a significant performance penalty.

A simpler solution would be to develop a special library implementing pattern matching functionality. This approach is followed for example in the AsF+SDF group [10] where a C library called ATERms 9] has been developed. In this library, pattern matching is implemented via a function called ATmatch, which consists in matching a term against a single pattern represented by a string or a term. Therefore, it is possible to define a transformation by pattern matching, thanks to a sequence of if-then-else instructions, where each condition is a call to the ATmatch function. But this approach has three drawbacks. First, matching is performed sequentially: patterns are tried one by one. This can be rather inefficient for a large number of patterns. Second, terms and patterns are untyped, and thus may be error prone. Third, the programming language and the data structure are imposed by the library, and so the programmer cannot use his favorite language as well as his own data structure to represent terms.

To solve the deficiencies of the above two solutions, and in particular for the sake of efficiency, we are interested in the compilation of pattern matching. By compilation we mean an approach where all patterns are compiled together producing a matching automaton. This automaton then performs matching against all patterns simultaneously. Our research on this topic is guided by the following concerns:

- How to efficiently compile different forms of pattern matching? We are concerned by simple syntactic matching but also by matching modulo an equational theory. In such a case, for example a pattern $x+3$ can match expression $3+7$ thanks to commutativity of plus.

- How to implement compilation of pattern matching in a uniform way for a large class of programming languages and for any representation of terms?

To tackle the above mentioned problems, we develop a non-intrusive pattern matching compiler called ToM. Its design follows our experiences on the efficient compilation of rule-based systems 311. Our tool can be viewed as a YACClike compiler translating patterns into executable pattern matching automata. Similarly to YACC, when a match is found, the corresponding "semantic action" (a sequence of instructions written in an imperative language) is triggered and executed. In a way, we can say that Tom translates a declarative-imperative function - defined by pattern matching and imperative instructions - into a fully imperative function. The resulting function can be integrated to an application 
written in a classical language such as C, Java, or Eiffel, called the target language in the rest of the document. In this paper, we illustrate the different advantages of the approach implemented by Tom, namely:

Efficiency. The gain of efficiency follows from the compilation of matching as implemented in ToM.

Flexibility. When trying to integrate a black-box tool in an existing system, one of the main bottlenecks comes from data conversion and the flexibility offered to the user. One of the main originalities of our system is its independence of term representation. The programmer can use (or re-use) his own data structures for terms/trees and then execute matching upon those data structures. We propose to access terms using only a simple Application Programming Interface (API) defined by the user.

Generality. TOM is able to consider multiple target languages (C, Java, and Eiffel). TOM is implemented in TOM itself as a series of AST transformations. The code generation is performed at the very end, depending on the target language we are interested in. Hence, the target language is really a parameter of TOM.

Expressivity. Tom supports non-linear patterns and equational matching like modern rule-based programming languages. Currently, we have implemented pattern matching with list operators. This form of associative matching with neutral element is very useful for practical applications. The main difference with standard (syntactic) matching is that a single variable may have multiple assignments.

The paper is organized as follows: Section 2 motivates the main features of TOM on a very simple example. In Section 3 , we present the main language constructs and their precise meanings. Further applications are described in Section 4. Since TOM is non-intrusive, it can be used in the context of existing applications to implement in a declarative way some functionalities which can be naturally expressed as transformations of terms (Section 4.1). Furthermore, we show how TOM is used in designing a compiler, via some transformations of ASTs performed by pattern matching: in fact, this example is the current implementation of TOM itself (Section 4.2). Section 5 presents some related work and Section [6] concludes with final remarks and future work.

\section{What Is Tom?}

In this section, we outline the main characteristics of TOM and we illustrate its usage on a very simple example specifying a well-known algebraic data type, namely the Naturals.

Tom does not really define a new language: it is rather a language extension which adds new matching primitives to an existing imperative language. From an implementation point of view, it is a compiler which accepts different native languages: C, Java, and Eiffel. The compilation process consists of translating new matching constructs into the underlying native language. Since the native 
language and the target language are identical and only introduced constructs are expanded, the presented tool can also be seen as a kind of preprocessor. On the other hand, the support of multiple target languages, and the fact that the input program has to be completely parsed before the transformation process can begin, make us consider ToM as a compiler.

For expository reasons, we assume that Tom only adds one new construct: $\%$ match. This construct is similar to the match primitive found in ML and related languages: given a term (called subject) and a list of pairs: pattern-action, the match primitive selects a pattern that matches the subject and performs the associated action. This construct may thus be seen as an extension of the classical switch/case construct. The main difference is that the discrimination occurs on a term and not on atomic values like characters or integers: the patterns are used to discriminate and retrieve information from an algebraic data structure.

To give a better understanding of ToM's features, let us consider a simple symbolic computation (addition) defined on Peano integers represented by zero and successor. When using Java as the native language, the sum of two integers can be described in the following way:

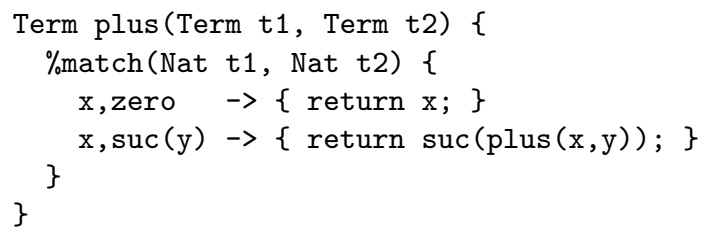

This example should be read as follows: given two terms $t_{1}$ and $t_{2}$ (that represent Peano integers), the evaluation of plus returns the sum of $t_{1}$ and $t_{2}$. This is implemented by pattern matching: $t_{1}$ is matched by $x, t_{2}$ is possibly matched by the two patterns zero and $\operatorname{suc}(y)$. When zero matches $t_{2}$, the result of the addition is $x$ (with $x=t_{1}$, instantiated by matching). When $\operatorname{suc}(y)$ matches $t_{2}$, this means that $t_{2}$ is rooted by a suc symbol: the subterm $y$ is added to $x$ and the successor of this number is returned. The definition of plus is given in a functional programming style, but the plus function can be used in Java to perform computations. This first example illustrates how the \%match construct can be used in conjunction with the considered native language.

In order to understand the choices we have made when designing ToM, it is important to consider TOM as a restricted compiler: it is not necessary to parse the native language in detail in order to be able to replace the \%match constructs by a sequence of native language instructions (Java in this example). This could be considered as a kind of island parsing, where only the ToM constructs are parsed in detail. The first phase of the transformation process consists of reading the program: during this phase, the text is read and Tom constructs are recognized, whereas remaining parts are considered as target language constructs. The output of this first phase is a tree which contains two kinds of nodes: target language nodes and Tom construct nodes. When applied to the previous example, we get the following program with a unique Tom node, represented by a box as follows: 


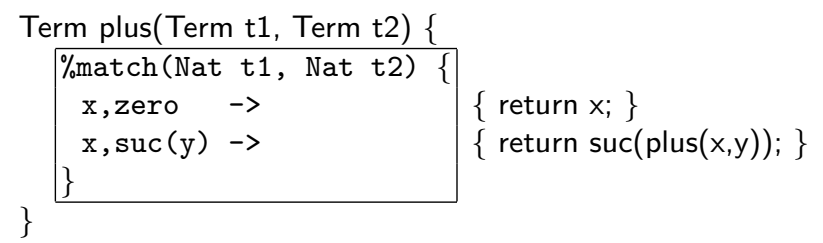

Tom never uses any semantic information of the target language nodes during the compilation process, it does not inspect nor modify the source language part. It only replaces the TOM constructs by instructions of the native language. In particular, the previous \%match construct will be replaced by two nested if-then-else constructs.

At this point, it is interesting to note that plus is a function which takes two Term data structures as arguments, whereas the matching construct is defined on the algebraic data type Nat. This remark introduces the second generic aspect of TOM: the matching can be performed on any data structure. For this purpose, the user has to define its term representation and the mapping between the concrete representation and the algebraic data type used in matching constructs.

To make our example complete, we have to define the term representation Term and the algebraic data type which defines the sort $N a t$ and three operators: $\{$ zero $: \mapsto N a t, \quad$ suc $:$ Nat $\mapsto$ Nat, plus : Nat $\times$ Nat $\mapsto N a t\}$

For simplicity, we consider in this example that the ATERM library [9] is used for term representation. This library is a concrete implementation of the Annotated Terms data type (ATERMs). In particular it defines an interface to create and manipulate term data structures. Furthermore, it offers the possibility to represent function symbols, to get the arity of such a symbol, to get the root symbol of a term, to get a given subterm, etc. The main characteristic of this library is to provide a garbage collector and to ensure maximal sharing of terms. Using this library, it becomes easy to give a concrete implementation of function symbols zero and suc (the second argument of makeAFun defines the arity of the operator): AFun $f_{-z e r o}=\operatorname{makeAFun}(" z e r o ", 0)$ and AFun $f_{-s u c}=$ makeAFun ("suc",1). The representation of the constant zero, for example, is given by makeAppl ( $f \_z e r o$ ). Similarly, given a Peano integer $t$, its successor can be built by makeAppl (f_suc,t). So far we have shown how to represent data using the ATERM library, and how defining matching with TOM, but, we have yet to reveal how these two notions are related. Given a Peano integer $t$ of sort Term, we have to define how to get its root symbol (using getAFun for example) and how to know if this symbol corresponds to the algebraic function symbol $s u c$, intuitively getAFun( $t$ ). isEqual (f_suc).

This mapping from the algebraic data type to the concrete implementation is done via the introduction of new primitives, \%op and \%typeterm, which are described in the next section.

\section{The Tom Language: Main Constructs}

In the previous section we introduced the match construct of TOM via an example. In this section, we give an in-depth presentation of ToM by explaining 
all existing constructs and their behavior. As mentioned previously, TOM introduces a new construct (\%match) which can be used by the programmer to decompose by pattern matching a tree-like data structure (an ATERM for example). Tom also introduces a second family of constructs which is used to define the mapping between the algebraic abstract data type and the concrete implementation. We distinguish two main constructs: \%typeterm and \%op are used to define respectively algebraic sorts and many-sorted signature of the algebraic constructors.

\subsection{Sort Definition}

In Tom, terms, variables, and patterns are many-sorted. Their algebraic sorts have to be introduced by the \%typeterm primitive. In addition to this primitive, the mapping from algebraic sorts to concrete sorts (the target language type, such as Term) has to be defined. Several sub-functions are used for this purpose. To support the intuition, let us consider again the Naturals example where the Nat algebraic sort is implemented by ATERMs. One possible mapping is the following:

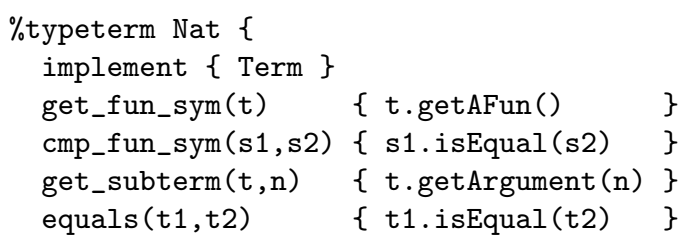

- The implement construct describes how the algebraic type is implemented. The target language part (written between braces: ' $\{$ ' and '\}') is never parsed, it is only used by the compiler to declare some functions and variables. This is analogous to the treatment of semantic actions in YACC.

Since in this example we focus our attention on the ATERM library, we implement the algebraic data type using the "implement \{ Term \}" construct. But, if we suppose that another data structure is used, "struct myTerm*" for example, the "implement \{ struct myTerm* \}" construct should be used to define the mapping.

- get_fun_sym $(t)$ denotes a function (parameterized by a term variable $t$ ) that should return the root symbol of the term referenced by $t$.

As in the $C$ preprocessor, the body of this definition is not parsed, but the formal parameter ( $t$ ) can be used in the body ( $t$.getAfun ( ) in our example).

- cmp_fun_sym (s1,s2) denotes a predicate (parameterized by two symbol variables $s_{1}$ and $s_{2}$ ). This predicate should return true if the symbols $s_{1}$ and $s_{2}$ are "equal". The true value should correspond to the built-in true value of the considered target language. (true in Java, and something different from 0 in $C$ for example).

- get_subterm $(t, n)$ denotes a function (parameterized by a term variable $t$ and an integer $n$ ). This function should return the $n$-th subterm of $t$. This 
construct of $\mathrm{C}$ and Java, the body is a list of pairs: pattern-action. The pattern is a list of terms (with free variables) which are matched against a list of terms that compose the subject. When the pattern matches the subject, the free variables are instantiated and the corresponding action is executed. Note that this is a hybrid language construct, mixing two formalisms: the patterns are written in a pure algebraic specification style using constructors and variables, whereas the action parts are directly written in the native language, using the variables introduced by the patterns. Since Tom has no knowledge of what is done inside an action, the action part should be written in such way that the function has the desired behavior. In our Peano example, the $\operatorname{suc}(\mathrm{plus}(\mathrm{x}, \mathrm{y})$ ) expression corresponds to a recursive call of the plus function while the suc function is supposed to build a successor. Note that this part has nothing to do with Tom: it only depends on the considered target language. The semantic of the \%match construct is as follows:

Matching: given a subject, the execution control is transferred to the first pattern that matches the subject. If no such pattern exists, the evaluation of the \%match construct is finished.

Selected pattern: given a pattern which matches the subject, the associated action is executed, using the free variables instantiated during the matching phase. If the execution control is transferred outside the \%match construct (by a goto, break, or return statement for example), the matching process ends. Otherwise, the execution control is transferred to the next patternaction whose pattern matches the subject.

End: when no more pattern matches the subject, the \%match construct ends, and the execution control is transferred to the next target language instruction.

\subsection{Making Terms}

In addition to sort definition, construction definition and matching constructs, TOM provides a mechanism to easily build ground terms over the defined signature. This mechanism, called back-quote (and written ' '), can be used in any target language block as a kind of escape mechanism. The syntax is simple: the back-quote is followed by a well-formed term written in prefix notation. The last closing parenthesis denotes the end of the escape mechanism.

Considering the previously defined addition function on Peano integers, the right-hand side could have been written ' $\operatorname{suc}(\mathrm{plus}(\mathrm{x}, \mathrm{y})$ ) and the construction of the suc node would have been done by TOM, using the make attribute introduced in Section 3.2

\subsection{Equational Matching}

An important feature of TOM is to support equational matching. In particular, list matching, also known as associative matching with neutral element. 
Since a list can be efficiently and naturally represented by a (head,tail) tuple, Tom provides an extra construct for defining associative data structures: the \%typelist primitive. When defining such a structure, three extra access functions have to be introduced: get_head, get_tail and is_empty:

- get_head (1) denotes a function parameterized by a list variable $l$ that should return the first element of the list $l$. When using the TermList data type, the definition is "get_head(1) \{ $1 . \operatorname{getHead}()\}$ ".

- get_tail(1) denotes a function parameterized by a list variable $l$ that should return the tail of the list $l$. Using ATERMs, it can be defined by "get_tail(I) $\{1$.getTail() $\}$ ".

- is_empty(1) denotes a predicate parameterized by a list variable $l$. This predicate should return true if the list $l$ contains no element. One more time, the mapping to ATERMS is obvious: "is_empty(1) $\{1$.isEmtpy() $\}$ ".

Similarly to the $\%$ op construct, TOM provides the \%oplist construct to define list operators. When using such a construct, the user has to specify how a list can be built. This is done via the two following functions:

- make_empty() should return an empty list. This object corresponds to the neutral element of the considered data structure.

- make_insert $(e, 1)$ should return a new list $l^{\prime}$ where the element $e$ is inserted at the head of the list $l$ (i.e. expressions equals (get_head ( $\left.l^{\prime}\right), e$ ) and equals (get_tail (l'), l) should be true).

One characteristic of list-matching is the possibility to return several matches. Thus, the semantic of the \%match construct has to be extended as follows:

Selected pattern: given a pattern which matches the subject, for each computed match, the list of free variables is instantiated and the action part is executed. If the execution control is transferred outside the \%match construct the matching process ends. Otherwise, another match is computed. When no more match is available, the execution control is transferred to the next pattern-action whose pattern matches the subject.

This principle can be used to implement a sorting algorithm using a conditional pattern matching definition. In the following, we consider an associative data structure List and an associative operator conc:

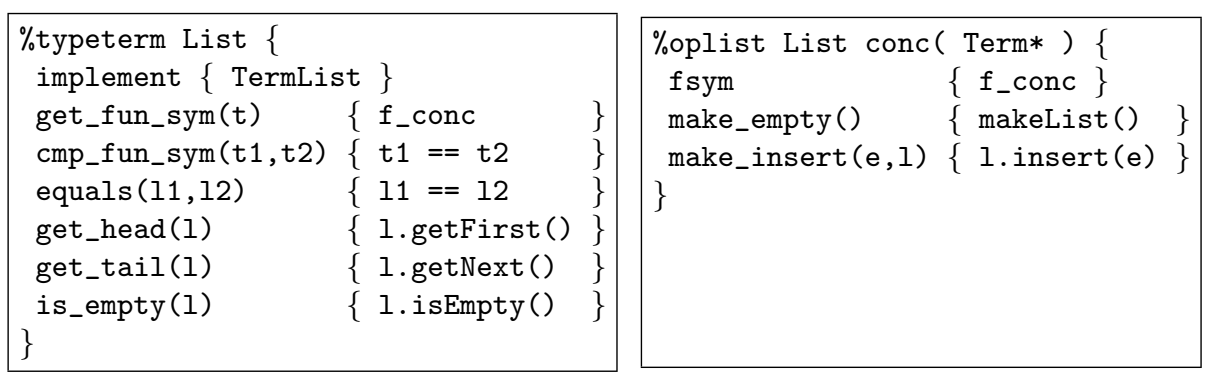




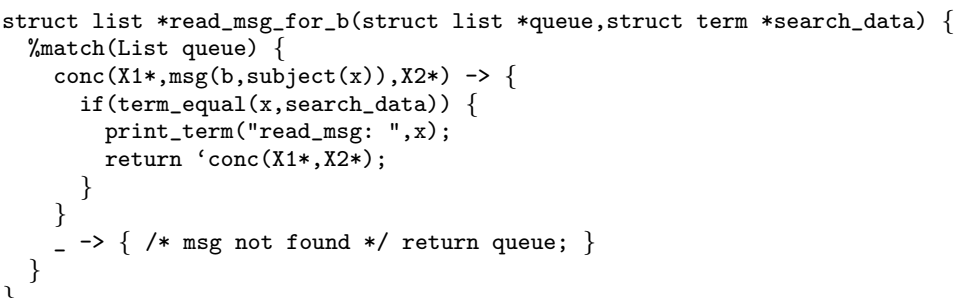

In this function, when a message addressed to $\mathrm{b}$ is found but does not correspond to search_data, another match is computed (all possible instances of $\mathrm{X} 1, \mathrm{x}$ and $\mathrm{X} 2$ are tried). If no match satisfies this condition, the default case is executed.

\subsection{Implementing Compilers and Transformation Tools}

The presented language extension has an implementation: jton 1 . One characteristic of this implementation is that it is written in TOM itself (Java+Tom to be more precise).

Compiling a program consists in transforming this program (written in some source language) into another equivalent program written in some target language. This transformation can be seen as a textual or syntactic transformation, but in general, this transformation should be done at a more abstract level to ensure the equivalence of the two programs. A good and well-known approach consists in performing the transformation of the AST that represents the program.

Representing an AST can be done in a "traditional way" by defining a data structure or a class (in an object oriented framework) for each kind of node. Another interesting approach consists in representing this tree by a term. Such an approach has several advantages. First, it is a universal representation for every manipulated information. Second, compared to a collection of spreaded objects in memory, a term can be more easily printed and exchanged with other tools at any stage of the transformation. Last, all the information is always available in the term itself.

Thus, given a program, its compilation can be seen as the transformation of a term (the AST of the source language program) into another term (the AST of the target language program). Transformation rules are usually expressed by pattern matching, which is exactly what Tom is suited for.

The implementation of the TOM compiler is an application of this principle: it is composed of several phases that respectively transform a term into another one. The general layout of the compiler is shown in Figure 1 .

As illustrated, four main compilation phases can be distinguished. Each phase corresponds to an abstract syntax whose signature is defined in ToM, using the signature definition formalism presented in Sections 3.1 and 3.2

1 available at http://elan.loria.fr/tom 


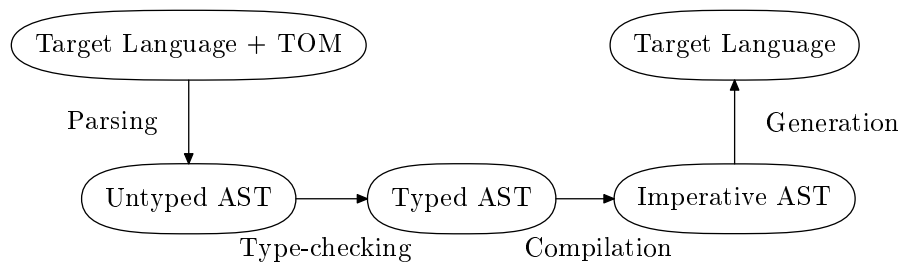

Fig. 1. General layout of the Tom compiler

Parsing. The Tom parser reads a program enriched by Tom constructs and generates an Abstract Syntax Tree. As mentioned previously, the source language is a superset of the target language, and we have the following particular equation: source language $=$ target language + TOM constructs .

In order to be as general as possible, the current TOM parser is only slightly dependent on the supported target languages. In particular, it does not include a full native language parser: it should only be able to parse comments, strings, and should recognize the beginning and the end of a block (' $\{$ ' and ' $\}$ ' in C or Java). Using this knowledge, the parser can detect and parse all ToM constructs. The resulting AST is a list of nodes of two kinds: (1) A Target Language Node is a string that contains a piece of code written in the target language. This node does not contain any Tom construct; (2) A Tom Construct Node is an AST that represents a TOM construct. The role of the TOM compiler consists in replacing all Tom Construct Nodes by new Target Language Nodes, without modifying, and even parsing, the remaining Target Language Nodes. When considering the Naturals example, after parsing, the pattern suc(y) is represented by the following AST:

Term (Appl (Name ("suc"), [Appl (Name ("y"), [])]))

Informally, this means that an operator called suc is applied to a list of subterms. This list is made of a unique term corresponding to the application of $\mathrm{y}$ to the empty list. At this current stage, it is not yet possible to know whether y is a variable or a constant. We can also remark that there is no type information.

Type-checking. For sake of simplicity, no type information is needed when writing a matching construct. In particular, TOM variables do not need to be declared, and the definition of the signature can appear anywhere. Consequently, any constant not declared in the signature naturally becomes a variable. Unfortunately, it makes the compilation process harder. During this phase, the TOM type-checker determines the type of each Tom construct and modifies the AST accordingly. The output formalism of this phase is a typed TOM AST as exemplified below:

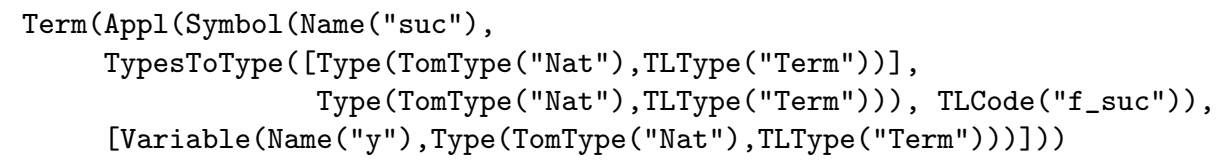


We can notice that the AST syntax (Term, Appl, Name, etc.) has been extended by several new constructors, such as Symbol, TypesToType, Variable, etc. A term corresponds now to the application of a symbol (and no longer a name) to a list of subterms. A symbol is defined by its name, its profile and its implementation in the target language ( $f_{-}$suc in this example). We can also notice that the profile contains two kinds of type information: the algebraic specification type (Nat) and the implementation of this type in the target language (Term).

Compilation. This phase is the kernel of the TOM compiler: it replaces all TOM constructs by a sequence of imperative programming language instructions. To remain independent of the target language, this transformation is performed at the abstract level: instead of generating concrete target language instructions, the compilation phase generates abstract instructions such as DeclareVariable, AssignVariable, IfThenElse. The output formalism also contains some abstract instructions to access the term data structure, such as GetFunctionSymbol, GetSubterm, etc. After compiling the previous term, we get the following AST (for a better readability, some parts have been removed and replaced by "..."):

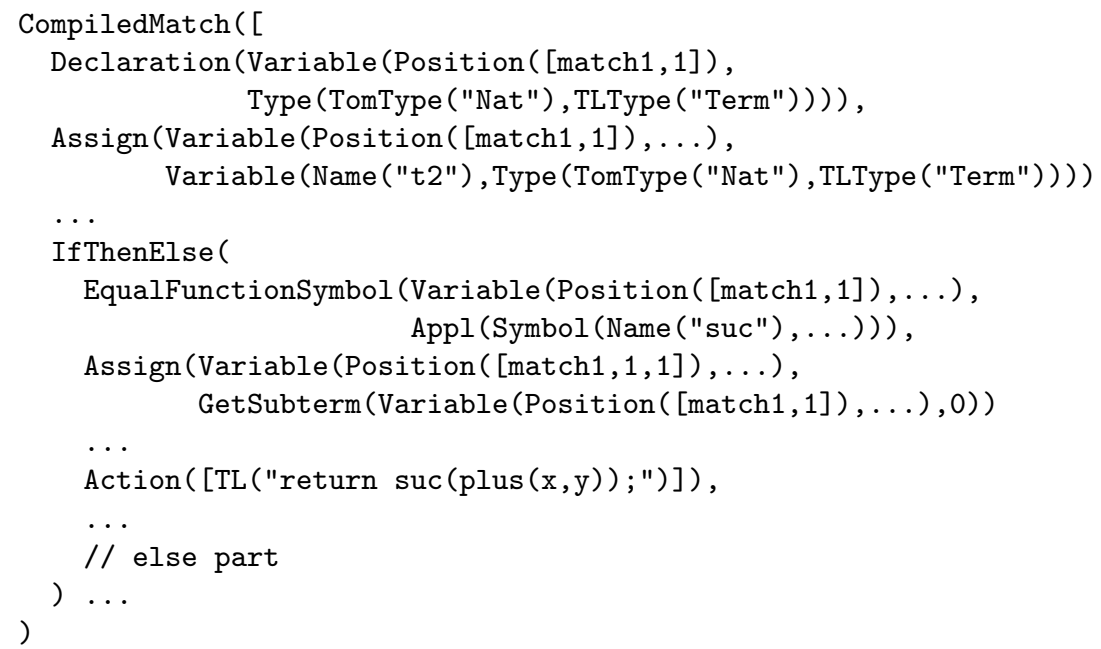

The main advantage of this approach is that the algorithm for compiling pattern matching does not depend on neither the target language nor the term data structure. During this phase, a match construct is analyzed, and depending on its structure, abstract instructions are generated (a Declaration and an Assignment when a variable is encountered for example, or a IfThenElse when a constructor is found for example).

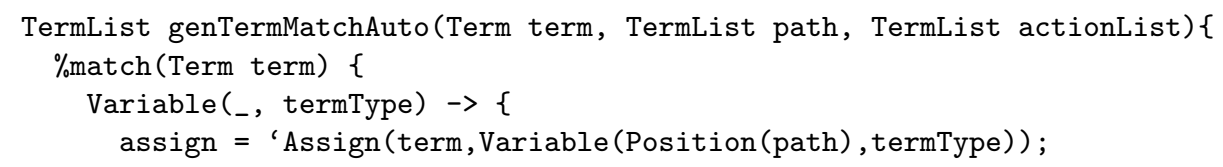




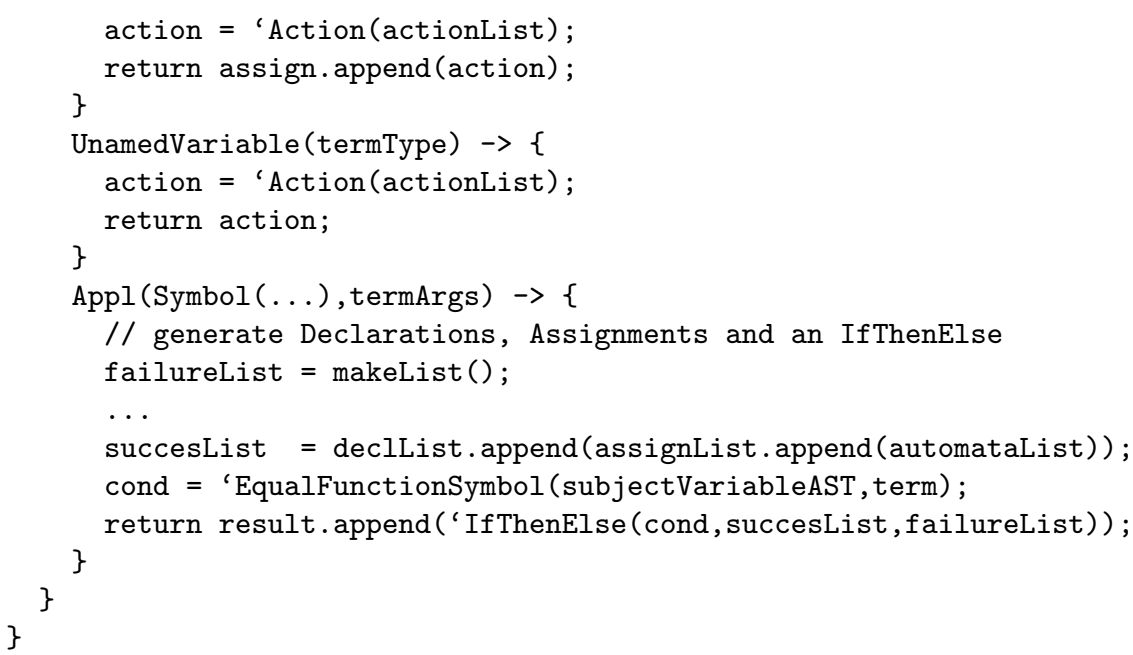

Generation. This phase corresponds to the back-end generator: it produces a program written in the target language. The mapping between the abstract imperative language and the concrete target language is implemented by pattern matching. To each abstract instruction corresponds a pattern, and an associated action which generates the correct sequence of target language instructions. In Java and $\mathrm{C}$ for example, the pattern-action associated to the IfThenElse abstract instruction is:

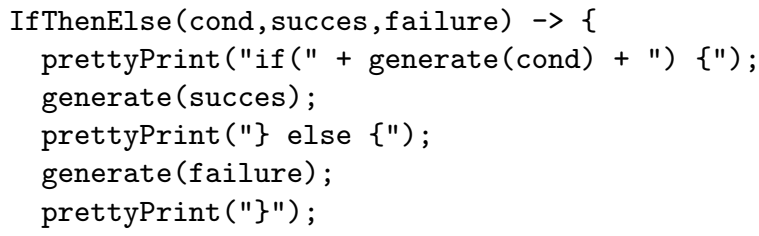

Due to lack of space, we cannot give much more detail about the compilation of ToM. But, our experience clearly shows that the main interests of TOM can be characterized by the expressiveness and the efficiency introduced by the powerful matching constructs. In practice, the use of pattern matching and list-matching helps the programmer to clearly express the algorithms and, as illustrated in the following table, it reduces the size of the programs by a factor 2 or 3 in average. We presents statistics for three typical ToM applications, corresponding to the three main components of the system: the type-checker, the compiler, and the generator. For each component, we report the self-compilation time in the last column (measured on a Pentium III, $1200 \mathrm{MHz}$ ). The first two columns give some size information. For instance, the type-checker consists of 555 lines including 40 pattern matching constructs. After being compiled, the generated Java code consists of 1484 lines. As illustrated by the compilation speed, the efficiency of the generated code is sufficient in practice for this kind of application. 


\begin{tabular}{|l|c|c|c|}
\hline Specification & $\begin{array}{c}\text { Tom } \\
\text { (patterns/lines) }\end{array}$ & $\begin{array}{c}\text { Generated Java code } \\
\text { (lines) }\end{array}$ & $\begin{array}{c}\text { Tom to Java } \\
\text { compilation time (s) }\end{array}$ \\
\hline \hline TOM checker & $40 / 555$ & 1484 & 0.331 \\
Tom compiler & $81 / 1490$ & 2833 & 0.600 \\
TOM generator & $87 / 1124$ & 3804 & 0.812 \\
\hline
\end{tabular}

\section{Related Work}

Several systems have been developed in order to integrate pattern matching and transformation facilities into imperative languages. For instance, $\mathrm{R}++[1]$ and App [7] are preprocessors for $\mathrm{C}++$ : the first one adds production rule constructs to $\mathrm{C}++$, whereas the second one extends $\mathrm{C}++$ with a match construct. Prop [4] is a multi-paradigm extension of $\mathrm{C}++$, including pattern matching constructs. Pizza [8] is a Java extension that supports parametric polymorphism, first-class functions, class cases and pattern matching. Finally, JaCo [13] is an extensible Java compiler written in an extension of itself: Java + extensible algebraic types.

All these approaches propose some very powerful constructs, but from our point of view, they are too powerful and less generic than ToM. In spirit, Prop, Pizza and JaCo are very close to ToM: they add pattern matching facilities to a classical imperative language, but the method of achieving this is completely different. Indeed, Prop, Pizza and JaCo are more intrusive than ToM: they really extend $\mathrm{C}++$ and Java with several new pattern matching constructions. On the one hand, the integration is better and more transparent. But on the other hand, the term data structure cannot be user-defined: the pattern matching process can only act on internal data structures. This may be a drawback when one wants to extend an existing project, since it is hard to convince a user to program in a declarative way if the first thing to do is to translate the existing main data structures.

\section{Conclusion and Further Work}

In this paper we have presented a non-intrusive tool for extending existing programming languages with pattern matching. In our opinion, TOM is a key component for the implementation of rule-based language compilers, as well as for the design of program transformation tools, provided that programs are represented by terms (using for instance ATERMS or XML representations). In this context, a prototype of ELAN compiler using TOM as back-end has already been successfully implemented for a subset of the language, and the ASF+SDF groun 2 and the ELAN groun 3 are currently designing a common extensible compiler based on TOM.

For the sake of expressiveness, it is important to continue the integration of equational matching into Tom. For now, we have successfully considered the

\footnotetext{
2 http://www.cwi.nl/projects/MetaEnv

3 http://elan.loria.fr
} 
case of list-matching, which was already supported by ASF+SDF. In the future, we still have to go beyond this first case-study by considering other more complicated and useful equational theories like Associativity-Commutativity and its extensions.

Acknowledgments. We would like to thank Mark van den Brand, Jurgen Vinju and Eelco Visser for fruitful discussions and comments on the design of TOM. We also thank the anonymous referees for valuable comments and suggestions that led to a substantial improvement of the paper.

\section{References}

1. J. M. Crawford, D. Dvorak, D. Litman, A. Mishra, and P. F. Patel-Schneider. Path-based rules in object-oriented programming. In Proceedings of the Eighth Innovative Applications of Artificial Intelligence Conference, pages 490-497, Menlo Park, 1996. AAAI Press / MIT Press.

2. P. Hudak, S. L. Peyton Jones, and P. Wadler (editors). Report on the Programming Language Haskell, A Non-strict Purely Functional Language (Version 1.2). SIGPLAN Notices, Mar, 1992.

3. H. Kirchner and P.-E. Moreau. Promoting rewriting to a programming language: A compiler for non-deterministic rewrite programs in associative-commutative theories. Journal of Functional Programming, 11(2):207-251, 2001.

4. L. Leung. Prop homepage: cs1.cs.nyu.edu/phd_students/leunga/prop.html.

5. J. Liu and A. C. Myers. Jmatch: Iterable abstract pattern matching for java. In V. Dahl and P. Wadler, editors, Proceedings of PADL'03, volume 2562 of LNCS, pages 110-127. Springer-Verlag, 2003.

6. R. Milner, M. Tofte, and R. Harper. The definition of Standard ML. MIT Press, 1990.

7. G. Nelan. App homepage: www.primenet.com/ ${ }^{\sim}$ georgen/app.html.

8. M. Odersky and P. Wadler. Pizza into Java: Translating theory into practice. In Proceedings of the 24th ACM Symposium on Principles of Programming Languages (POPL'97), Paris, France, pages 146-159. ACM Press, USA, 1997.

9. M. G. J. van den Brand, H. A. de Jong, P. Klint, and P. Olivier. Efficient annotated terms. Software-Practice and Experience, 30:259-291, 2000.

10. M. G. J. van den Brand, P. Klint, and P. Olivier. Compilation and Memory Management for ASF+SDF. In Proceedings of Compiler Construction, 8th International Conference, volume 1575 of LNCS, pages 198-213. Springer, 1999.

11. M. Vittek. A compiler for nondeterministic term rewriting systems. In H. Ganzinger, editor, Proceedings of RTA'96, volume 1103 of LNCS, pages 154-168, New Brunswick (New Jersey), 1996. Springer-Verlag.

12. P. Weis, M. Aponte, A. Laville, M. Mauny, and A. Suárez. The CAML Reference Manual. INRIA-ENS, 1987.

13. M. Zenger and M. Odersky. Extensible algebraic datatypes with defaults. In Proceedings of the 6th ACM SIGPLAN International Conference on functional Programming (ICFP'2001), Florence, Italy, pages 241-252. ACM Press, 2001. 